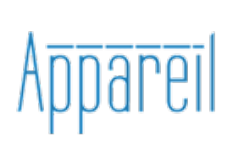

Appareil

4 | 2010

Biopolitique, éthique et subjectivation, questions de modernité

\title{
Pouvoir pastoral et « vie bête »
}

\section{Alain Brossat}

\section{(2) OpenEdition}

Journals

Édition électronique

URL : https://journals.openedition.org/appareil/898

DOI : 10.4000/appareil.898

ISSN : 2101-0714

Éditeur

MSH Paris Nord

Référence électronique

Alain Brossat, « Pouvoir pastoral et « vie bête » », Appareil [En ligne], 4 | 2010, mis en ligne le 27 janvier 2010, consulté le 12 octobre 2021. URL : http://journals.openedition.org/appareil/898 ; DOI : https:// doi.org/10.4000/appareil.898

Ce document a été généré automatiquement le 12 octobre 2021.

\section{(c) (†)}

Appareil est mis à disposition selon les termes de la Licence Creative Commons Attribution - Pas d'Utilisation Commerciale - Pas de Modification 4.0 International. 


\title{
Pouvoir pastoral et « vie bête »
}

\author{
Alain Brossat
}

1 À l'origine de ce texte, un étonnement: lorsque Foucault définit le pouvoir pastoral comme un grande forme (Omnes et singulatim, STP...), il en parle toujours exclusivement, jusqu'à ce qu'il aborde dans le détail la question du pastorat chrétien, et encore, pas dans les formes premières de celui-ci, du point de vue du pasteur, de son action, des motifs et principes qui guident son action (donc en faisant apparaître la double dimension de son "éthique » et de sa subjectivité ou des modes de subjectivation qui lui sont propres de son exercice du pouvoir), mais jamais du troupeau, des brebis. Le troupeau, les brebis sont constamment et exclusivement approchés comme le pur et simple objet de la conduite pastorale, du souci du pasteur. C'est-à-dire que l'imagerie animale est, littéralement, prise au pied de la lettre : le troupeau ne parle pas, les brebis sont dépourvues de la capacité d'interagir, en tant qu'élément " gouverné » ou conduit avec les desseins, les actions du pasteur pour la bonne et simple raison que leur condition reste inscrite dans la dimension de l'animalité - tout se passant comme si, dans la forme primitive ou plutôt première du pouvoir pastoral, les hommes étaient gouvernés à l'égal d'animaux, étant donné qu'aucune forme de subjectivité, aucune espèce d'accès au langage, aucune capacité de développer des contre-conduites n'apparaît du côté du «troupeau ». On peut même se demander si, pour Foucault, on parlerait dans ce topos, de relations entre gouvernants et gouvernés. Les brebis sont " conduites", plus que gouvernées, puisque, chez Foucault, constamment, la relation entre gouvernants et gouvernés, quelle qu'en soit la forme, suppose des interactions et quelque chose comme une circulation entre exercice du pouvoir consistant à tenter de «faire faire », à agencer des conduites, d'une part, et, de l'autre, des contre-conduites, des résistances, des paroles, des actions qui manifestent l'existence d'un véritable « contre-champ » du côté des gouvernés.

Or, ici, rien de semblable. Je rappelle très synthétiquement, la présentation que fait Foucault du pouvoir pastoral dans ses fondements et ses principes: à l'origine, le pastorat, c'est un rapport entre Dieu et les hommes. Une structure très ancienne qu'on trouve plutôt chez les Assyriens, les Égyptiens, les Hébreux que chez les Grecs. Dans ces civilisations, dit Foucault, les relations de Dieu avec son peuple sont définies comme des relations d'un pasteur avec son troupeau. Par transposition, cette structure 
pastorale du rapport entre Dieu et les hommes devient un modèle de pouvoir, dans la relation entre un guide-berger humain et un peuple ou une population.

Foucault insiste sur les caractéristiques propres de cette forme de pouvoir : elle n'est ni répressive ni autoritaire, le berger ne règne pas, comme un roi grec, sur un territoire ou une cité, il conduit, guide le troupeau humain, veille sur lui, prend garde à ce qu'il ne s'égare ni ne dépérisse. Il s'agit d'un pouvoir de soin: le berger ne gouverne pas " pour lui-même ", mais au contraire, entièrement "pour les autres ", le troupeau et ce sur un mode non seulement global, mais individualisant : tout son soin va au troupeau, mais, selon la célèbre image biblique, le berger veille à ce que chacune des bêtes qui le compose ne s'égare ni ne s'affaiblisse, au point de s'imposer d'abandonner provisoirement le troupeau pour retrouver et sauver une brebis égarée.

4 Tous les développements que consacre Foucault à cette forme première du pastorat sont voués à la description de ce que l'on pourrait appeler le cahier des charges du berger. Le troupeau n'a même pas d'existence propre: il «existe par la présence immédiate et l'action directe du pasteur " $(O$ et $S$ ). Que ce soit dans $O$ et $S$ ou bien dans STP, toute la description que fait Foucault de cette grande forme de pouvoir dans sa forme originale est exclusivement vouée à la figure du pasteur qui rassemble, guide, conduit son troupeau, en assure le salut, pratique à son égard une bienveillance constante et individualisée, connaît pour cette raison le troupeau dans l'ensemble et en détail, etc. Et il insiste tout autant sur le trait décisif, inaugural de l'apparition de cette forme :

Les sociétés qui sont apparues à la fin de l'Antiquité sur le versant occidental du continent européen ont inventé un grand nombre de formes politiques différentes [...] Mais elles seules ont développé une étrange technologie du pouvoir traitant l'immense majorité des hommes en troupeau avec une poignée de pasteurs ( 0 et $S$ ).

Deux étonnements à propos de cette première partie de la présentation du pouvoir pastoral.

6 Premièrement, dans les deux textes, et au rebours d'un certain sens commun, Foucault insiste sur cette spécificité du pouvoir pastoral - son trait individualisant - un pouvoir individuellement bienfaisant. D'autant plus surprenante est donc la complète absence d'une prise en compte de ce que serait la «brebis individuelle», dans son individualité propre. Comment cette prise en charge, ce «dévouement", peuvent-ils se manifester en l'absence de tout échange, de toute interaction entre le berger et cette singularité ? Comment une telle relation établie entre, disons, la brebis égarée, la brebis malade, la brebis récalcitrante et le berger peut-elle se maintenir en deçà du langage ? Le " jeu » individualisant du pasteur va-t-il se limiter à identifier la brebis qui a " un problème » particulier à des traits physiques, un parcours erratique ? Comment prendre en charge ce problème sans que s'établisse quelque chose comme une relation singulière ? Sur ce point, rien dans le texte de Foucault.

7 Deuxièmement, il suffit d'ouvrir la Bible, à laquelle Foucault fait référence constamment dans ces textes pour s'apercevoir que, précisément, le "troupeau " humain y a une « présence » propre, une capacité de se manifester qui le définit comme le pur objet du soin du pasteur. Assurément, si l'on prend un texte comme L'Exode, il y est question, avant tout, de la façon dont va s'établir la relation verticale entre les Hébreux comme peuple et leur Dieu, Moïse se voyant assigner, dans ce parcours, le rôle redoutable d'intermédiaire, de mandataire auprès du peuple de la puissance divine (l'Éternel). La «structure pastorale » évoquée par Foucault est donc bien là, le Dieu 
hébraïque conduit bien son troupeau vivant à travers le désert, il est bien une présence souvent visible, il guide, il montre, il rassure, il exhorte... Mais, contrairement à ce qui est le cas dans l'analyse foucaldienne, le troupeau est aussi un peuple : il manifeste, pour le meilleur et pour le pire, des dispositions collectives, il a ses mouvements propres qui, souvent, contreviennent aux directives émanant de leur Dieu et qui lui sont ponctuellement transmises par Moïse. Un peuple qui, souvent, prend la parole pour dire son découragement, sa colère, son impatience, ses frustrations, qui conteste les injonctions transmises par Moïse, qui doute, qui erre et s'éloigne des commandements tout au long de ce parcours initiatique que constitue la fuite hors d'Égypte - voir le fameux épisode du Veau d'or.

8 Il apparaît donc ici que la forme pastorale, dans sa forme primitive, n'est nullement incompatible avec la présence d'un troupeau non seulement défini comme vivant collectif, mais comme parlant et doté de capacités subjectives propres: esclaves de Pharaon, les Hébreux demeurent un ensemble vivant dans les ténèbres, n'ayant pas encore scellé l'Alliance avec son Dieu qui le fera accéder à son génie propre - mais ils ne sont pas pour autant réductibles à la condition d'une animalité muette et impensante : ils sont un peuple égaré et maltraité, mais un peuple humain. Et leur existence comme telle n'est pas réductible à ce à quoi la mission de Moïse les destine : être le Peuple de Dieu.

9 Foucault s'appuie, dans 0 et $S$, sur une minutieuse analyse du Politique de Platon pour montrer que la forme de pouvoir pastorale est, pour l'essentiel, étrangère à la pensée des Grecs anciens - ce serait le sens de la démonstration à laquelle Platon procède dans ce dialogue et au fil de laquelle il invaliderait la figure du berger, pasteur d'hommes, au profit de celle du tisserand. Il rappelle l'argumentation de Platon disant que ce n'est pas en distinguant la conduite des animaux de celle des hommes (en prenant en compte l'espèce qui est commandée) que l'on peut approcher de façon satisfaisante une définition de la forme de pouvoir qui s'exerce dans la cité - ce n'est pas, pour Platon, en décidant quelles espèces peuvent former un troupeau, mais en analysant ce que fait le berger, insiste Foucault, que l'on peut dire si le roi est ou non une sorte de pasteur. Une remarque qui éclaire l'«impasse» faite par Foucault sur l'existence subjective et les dispositions du troupeau, animal ou humain, cette distinction s'avérant sans importance tant l'analyse de la figure pastorale en sa forme originaire est intégralement et sans reste concentrée sur le rôle et la fonction du berger, comme si cette forme de pouvoir s'exerçait sur un mode totalement asymétrique, dans la relation unilatérale entre un sujet et un objet (vivant). À l'évidence alors, cette figure représenterait une sorte d'exception (notable) aux conditions générales de l'analytique du pouvoir présentée par Foucault, dans La Volonté de savoir, par exemple, où l'accent est constamment placé sur la liberté des gouvernés et sur leur capacité de prendre le pouvoir à son propre jeu en produisant des formes de contre-pouvoir. Sur ce point, aucune réponse n'apparaît dans les textes sur lesquels nous nous appuyons ici.

Dans Le Politique, le pouvoir pastoral est défini, rappelle Foucault, comme un pouvoir de prise en charge intégrale par le berger, seul à la tête de son troupeau, du soin de la vie de celui-ci : il veille à le nourrir, à le soigner, à l'encadrer, à aider sa reproduction, à le distraire, même... Une sorte de biopolitique totale avant la lettre, donc. Or, insiste Platon, il est de notoriété publique que, dans la Cité grecque, la multitude des fonctions ici évoquées n'échoient pas à un seul homme, (peut importe la façon dont on le définit le dirigeant, le roi, l'homme politique), mais à une quantité de sujets - le médecin, le 
boulanger, le cultivateur, le musicien, etc. La tâche de l'homme politique n'y est pas d'assumer, en pasteur, la totalité de ces fonctions d'entretien, mais d'associer des tempéraments divers, de rassembler les vivants « en une communauté qui repose sur la concorde et l'amitié »-d'où l'image qui s'impose ici, celle du tissage de tissus de différentes matières et couleurs. Ni Dieu ni berger, le politique a pour tâche d'assurer l'unité de la cité, une tâche, pourrait-on ajouter, qui suffit amplement à sa peine.

11 Mais ce qui est intéressant ici, c'est que, pour Foucault, un pastorat intégralisé, comme figure de l'exercice du pouvoir fondé sur la prise en charge globale et multipolaire des aspects de la vie du troupeau ne semble toujours pas davantage supposer autre chose que la compétence supérieure d'un berger avisé - un Dieu, dit-il, ce qui exclut cette forme pour la Cité qui se trouve être peuplée et dirigée par des hommes exclusivement. Même dans l'hypothèse (rejetée) du pastorat transposé des espaces orientaux vers les topographies européennes et l'espace politique par excellence - la Cité - la question de la condition subjective du troupeau n'est pas posée - or comment imaginer la possibilité d'un tel gouvernement multidirectionnel du troupeau humain sans que celui-ci en soit, d'une manière ou d'une autre partie prenante, sans qu'il y soit présent autrement que dans la condition d'un gros animal bien ou mal traité, mais indéfiniment muet?

12 Tout va donc se passer comme si, dans l'analyse de Foucault, le troupeau mutique et sans subjectivité, le troupeau non pas «vie nue», mais "vie bête» allait se transfigurer, changer totalement de qualité à la faveur d'une sorte de coup de théâtre, à mon sens assez difficilement explicable - celui qui se produirait avec la captation et le redéploiement de la grande forme pastorale dans et par le christianisme. Tout se passe au fond comme si une subjectivité un accès au langage venaient au troupeau, devenaient des attributs des brebis, dès lors que le coup de baguette magique de la pastorale chrétienne allait transfigurer cette grande forme de pouvoir venue du fond des temps, du lointain des civilisations orientales. Le paradoxe de cette métamorphose est fort: c'est bien, si l'on suit Foucault, dans des conditions où il entre dans une "relation de dépendance individuelle et complète", où se trouve établi entre lui et son guide spirituel un « lien de soumission personnelle ", d'obéissance absolue, qu'il va accéder à la parole et devenir un sujet. En effet, les formes de l'individualisation du pastorat chrétien supposent que le berger (le prêtre) ne se contente pas de guider et encadrer des formes extérieures, mais qu'il entre dans l'espace des subjectivités - il doit « savoir ce qui se passe dans l'âme de chaque membre du troupeau ». Et pour que ce nouveau pan de la conduite des brebis puisse s'établir, il faut que soient prises en compte les subjectivités (le désir, les sentiments, les dispositions, les pensées) et que des récits soient agencés par les sujets conduits eux-mêmes.

13 Le pivot de ce retournement va donc être ce double dispositif mis en place par l'Église chrétienne : l'examen de conscience et la direction de conscience - avec son débouché, la confession. L'aveu va se trouver installé au centre de tout un dispositif de subjectivation, de tout un dispositif de prise de parole par lequel se manifeste l'émergence du troupeau comme acteur à part entière de la grande prosopopée du pouvoir. Aux conditions de ce dispositif unique, sans équivalent dans l'histoire des civilisations humaines, grandiose et terrifiant tout à la fois, le troupeau n'accède pas seulement au discours et à des formes de subjectivation infiniment variables - il entre dans l'espace d'un «jeu »,dit Foucault, qui l'institue comme l'autre pôle, à part entière, de la machinerie du pouvoir : un « étrange jeu dont les éléments sont la vie, la mort, la 
vérité, l'obédience, les individus, l'identité ". Une étrange combinaison va alors se produire entre les réquisits fondamentaux du pastorat - le troupeau est là pour être guidé et sa disposition à être conduit (à l'obéissance et la docilité, donc) est, de ce point de vue, inscrite dans sa nature - et les dynamiques propres à l'œuvre dans le pastorat chrétien dont le propre est de constituer, dans la relation entre le conducteur et le conduit, l'espace d'une réflexivité et un théâtre de l'échange. Une "zone grise » s'établit entre ce qui tend à reconduire les brebis aux conditions premières et structurelles du pastorat - le caractère essentiellement, violemment asymétrique de la relation entre un conducteur divin ou humain et un « conduit » humain ou animal - et l'accès du troupeau à une forme d'autoréflexivité, morale notamment, qui le rend, au fond, disponible pour toutes sortes de mouvements de décentrement, si ce n'est d'échappée hors des conditions mêmes du pastorat. L'idée de Foucault, c'est que cet état d'indistinction entre l'un et l'autre pôle qui constitue le trait particulier de ce qu'il appelle les pouvoirs modernes en Occident - ce qui fait que ceux-ci vont pouvoir, par exemple, "apparaître " aussi bien sous la forme de monstrueuses entreprises de conduite du troupeau humain vers l'abîme des embrigadements massifs débouchant sur toutes sortes de destructions ou de désastres que sous celle de la promotion d'un « sujet » qui, ayant accédé à cette condition de réflexivité, est en mesure de s'arracher à son immémoriale condition d'hétéronomie.

Nous touchons là du doigt cette sorte de condition d'incertitude ou de part d'indétermination qui est le propre de la biopolitique contemporaine. D'une certaine façon, en effet, celle-ci tend à se rapprocher du modèle que Platon écarte, dans Le Politique, en faveur de celui du royal tisserand : une prise en charge par un pasteur aux mille visages, mais agissant de manière coordonnée, intégrée (donc une sorte de «dieu » immanent à la vie de l'État et de la société) de tous les aspects possibles et imaginables de la vie du troupeau humain. À l'évidence, dans nos sociétés, la prise en charge de la vie est non pas le fait de corporations différentes et spécialisées, agissant indépendamment les unes des autres et chacune pour son propre compte, mais bien un mécanisme général, intégré et différencié. Et, comme l'énonce Foucault dans La Volonté de savoir, c'est bien cette forme de la biopolitique, du biopouvoir qui, dans les sociétés modernes, a refoulé ou surdéterminé les formes traditionnelles indexées sur la souveraineté classique ou l'idéal de la communauté entée sur une axiologie partagée par ses membres. Et donc, plus que jamais, la relation entre gouvernants et gouvernés apparaît comme indexée sur la grande forme pastorale et ce n'est pas pour rien que les questions sanitaires et sécuritaires (celles qui ont trait à l'immunisation et l'entretien des corps) s'imposent comme l'objet majeur du gouvernement contemporain des vivants, au détriment notamment des formes classiques de la vie politique indexées sur la mise en forme et l'institutionnalisation des conflits. La prolifération des images médicales et policières dans le vocabulaire des gouvernants aujourd'hui est un autre indice de la surdétermination de toutes les rationalités et de tous les dispositifs politiques et autres agencements de pouvoir par la forme pastorale adaptées aux conditions de la modernité tardive.

Mais en même temps, le paradoxe de ce pastorat contemporain est le suivant : plus se poursuit sans relâche son mouvement de globalisation, de mondialisation, d'« intégralisation », et plus il apparaît qu'il « fuit » par un autre bout. C'est, si l'on veut rester fidèle à l'hypothèse foucaldienne, que le christianisme est passé par là et a laissé une empreinte ineffaçable sur la constitution des sujets dans leurs rapports avec le pouvoir, dans la façon dont ils entrent dans l'espace des relations de pouvoir. De la 
même façon que, dans le dispositif de la confession, s'établit une relation à peu près indiscernable entre soumission et constitution d'un " espace propre ", d'une condition d'intériorité susceptible d'agir comme propédeutique de la liberté, de la même façon, va se former, dans les espaces biopolitiques contemporains, ce cercle énigmatique : plus le pastorat global étend son emprise, améliore ses techniques et les diversifie, plus donc se démultiplient les procédures d' «objectivation» du troupeau et de ses conduites, plus sont nombreux et différenciés les messages qui lui sont adressés par le conducteur anonyme, et plus, par ailleurs, se trouvent démultipliées pour lui les occasions de rétroagir et de former des espaces discursifs dans lesquels la conduite pastorale se trouve, si l'on veut, prise dans son contrechamp. De la même façon que l'examen de conscience et la confession supposent qu'un espace soit ouvert aux facultés discursives des ouailles, dans la biopolitique contemporaine, la normation disciplinaire ne suffit plus : d'une manière toujours croissante, la bonne "gouvernance " biopolitique en appelle au discernement des sujets conduits, à leur responsabilité, à leur capacité à se projeter dans l'avenir. On gouverne désormais moins aux disciplines qu'aux mécanismes de sécurité et à la quête de l'assentiment. Apparaissent donc en permanence ces «lignes de fuite » hors de conditions premières du pouvoir pastoral, puisque là où s'ouvrent ces espaces dans lesquels les « conduits » sont promus (sinon à proprement parler institués) comme sujets, où il est fait appel à leurs facultés discursives, il peut arriver - et il arrive constamment - qu'au lieu de consentir et donner leur assentiment ils se rebiffent, qu'au lieu de "comprendre» ce qu'on leur "explique" ils forment leurs propres raisonnements, qu'au lieu de marcher en troupeau, ils se dispersent, et, sans même entrer en résistance ouverte, deviennent, de ce fait même, ingouvernables. De ce point de vue, on constate aisément que les retours de la politique dans sa forme la plus " classique » - la division descendue et exposée sur la place publique, au cœur même des formes biopolitiques - est un phénomène des plus courants dans nos sociétés : il n'y a pas si longtemps qu'ayant élu fraîchement un nouveau président de teinte fort conservatrice, les Sud-Coréens n'ont pas tardé à descendre par dizaines de milliers dans la rue, dès lors que celui-ci s'est avisé d'autoriser à nouveau l'importation de viande de bœuf des États-Unis, au mépris des avis de l'autorité sanitaire...

16 Mais, ceci ayant été dit, à l'encontre des discours catastrophiques qui annoncent chaque matin la «mort de la politique » au profit de la pure et simple prolifération des technologies destinées à assurer l'«apprivoisement», la domestication ou l'asservissement $d u$ troupeau humain, il conviendra de procéder à un ultime retournement. Celui-ci pourrait consister à s'aviser que la façon avec laquelle Foucault définit ce que l'on pourrait appeler le "pastorat fondamental » dans $O$ et $S$ ou STP est apparemment si brutale, si simplificatrice, en présentant cette relation si violemment asymétrique entre un pasteur, ni roi ni souverain, mais assurément dépositaire exclusif de l'intelligence de la relation de pouvoir établie entre le berger et ses ouailles, que cette absolue disparité et différence de qualité entre le guide et le guidé se retrouve, fait retour, indéfiniment, dans toute espèce de forme pastorale, de pouvoir pastoral et ce aussi "sophistiqué» soit-il. Et ceci non pas à l'état de trace, de séquelle, mais bien d'élément structurel ou structurant. De ce point de vue, il est essentiel de se rappeler que, contrairement à ce qu'en dit Platon, l'enjeu de l'hétérogénéité entre condition d'humanité et condition d'animalité ou plutôt entre statut (qualité) humaine et absence de qualité animale demeure ici constant. Dans toute forme de pastorat, antique, moderne ou contemporain, la qualité humaine est toute entière concentrée du côté du 
guide. Et s'il importe peu, au fond, que les guidés soient des animaux ou des humains, c'est précisément que dans la relation pastorale elle-même, quelque chose d'essentiel les reconduit à l'animalité, à la « vie bête » (par opposition, encore une fois, à la vie nue de Arendt revisitée par Agamben). Ce «quelque chose», on peut le nommer de toutes sortes de façons, la plus simple et la plus éclairante étant sans doute celle qui consiste à rappeler que la forme pastorale exclut absolument tout principe ou toute procédure d'égalisation entre guides et guidés ; ceci pour la bonne raison que le ou les savoir(s) de la conduite demeure(nt), en toutes circonstances, rigoureusement hétérogènes au savoir requis pour être guidé, au savoir et à la compréhension que les guides attendent du côté des guidés, afin que ceux-ci soient susceptibles de l'être - guidables. Dans la langue corrompue de la "gouvernance " contemporaine, cette différence de statut radicale entre l'intelligence supposée des guides et celle des guidés se manifeste dans l'envahissement du discours des premiers par les paradigmes pédagogiques : le troupeau humain est une classe (au sens scolaire du terme) rétive et distraite à laquelle il convient d'expliquer, sans fin, en termes simplifiés, ce qu'il est nécessaire qu'il sache afin de demeurer guidable. En aucun cas, le savoir des guidés ne saurait s'égaler à celui des pasteurs et, quand bien même cela serait imaginable, ce n'est évidemment pas souhaitable en termes de police sociale. La pédagogie "politique ", celle que requiert une sage conduite du troupeau, consiste à ne rendre disponible à celui-ci que la somme de savoirs et d'informations nécessaires pour que celui-ci demeure susceptible d'être conduit. Ici, une fois encore, les images médicales sont probantes: tout comme le médecin n'expose au patient que ce qui est nécessaire pour que celui-ci suive le traitement nécessaire pour soigner l'affection dont il souffre, en évitant de se lancer dans un cours de médecine, le politique s'efforcera de ne diffuser après du public que des messages "utiles » et simplifiés, rassurants plutôt que véridiques, à propos de la crise financière qui secoue le monde (octobre 2008). C'est que la faculté de discernement à laquelle il est fait appel, du côté des guidés, persiste à être, pour les guides, celle d'animaux intelligents. Ces animaux intelligents sont ce que, d'un autre côté, nous appelons « la vie bête ", c'est-à-dire, fondamentalement la vie qui ne peut prendre forme qu'aux conditions du pastorat, qu'à condition d'être guidée.

17 En ce sens, ce qui, au début de cet exposé, m'est apparu comme une sorte de bévue de Foucault pourrait bien être, plutôt, un effet aveuglant de son art de philosopher à coup de marteau, c'est-à-dire d'énoncer, souvent, des propositions proprement renversantes : en l'occurrence, celle qui consisterait à rappeler, en toute simplicité, que le propre d'une biopolitique moderne est d'établir, sur le long cours, une fondamentale condition d'indistinction entre troupeau humain et troupeau animal. Nous nous en doutions déjà un peu depuis que l'on a entrepris de nous convaincre qu'en tant que vivants, les animaux n'ont pas moins accès au droit à la vie que nous. Dès l'instant où le pivot de toute politique devient, au fond, la défense et la promotion du vivant, le trait inavouable d'un pastorat moderne revient en force: seule l'humanité des pasteurs est assurée, le troupeau est, lui, forme et matériau vivant avant tout, aux qualifications infiniment variables. 


\section{AUTEUR}

\section{ALAIN BROSSAT}

Département de philosophie, université Paris 8, abrossat@club-internet.fr 